\title{
Hedyotis diffusa Willd inhibits proliferation and induces apoptosis of 5-FU resistant colorectal cancer cells by regulating the PI3K/AKT signaling pathway
}

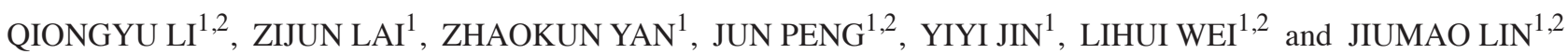 \\ ${ }^{1}$ Academy of Integrative Medicine; ${ }^{2}$ Fujian Key Laboratory of Integrative Medicine on Geriatrics, \\ Fujian University of Traditional Chinese Medicine, Fuzhou, Fujian 350122, P.R. China
}

Received November 9, 2016; Accepted July 20, 2017

DOI: $10.3892 / \mathrm{mmr} .2017 .7903$

\begin{abstract}
Hedyotis diffusa Willd (HDW) is a major component frequently used in Traditional Chinese Medicine for the clinical treatment of colorectal cancer (CRC) and its associated drug resistance. However, the underlying mechanism of HDW circumventing drug resistance of cancer cells remains to be elucidated. Cancer cell resistance to apoptosis and activation of the phosphatidylinositol-3-kinase (PI3K)/protein kinase B (AKT) signaling pathway have been implicated as major factors in the acquired resistance to chemotherapeutic anti-cancer drugs. The present study investigated the effect and mechanisms of action of ethanol extract of Hedyotis diffusa Willd (EEHDW) on the proliferation and apoptosis of CRC 5-fluorouracil (5-FU) resistant (HCT-8/5-FU) cells. CRC HCT-8/5-FU cell viability following treatment with EEHDW was determined using MTT and colony formation assay. In addition, Annexin $\mathrm{V} /$ propidium iodide staining with flow cytometry analysis and 4',6-diamidino-2-phenylindole staining were used to determine the apoptosis of drug-resistant cancer cells following treatment with EEHDW. The mRNA and protein expression levels of B cell leukemia/lymphoma (Bcl-2), Bcl-2 associated X (Bax), cyclin dependent kinase 4 (CDK4), cyclin D1 and p21 were evaluated using reverse transcription-polymerase chain reaction and western blot analysis, respectively. Furthermore, activation of
\end{abstract}

Correspondence to: Professor Jiumao Lin, Academy of Integrative Medicine, Fujian University of Traditional Chinese Medicine, 1 Qiuyang Road, Minhou Shangjie, Fuzhou, Fujian 350122, P.R. China

E-mail: jiumaolin@hotmail.com

Abbreviations: EEHDW, ethanol extract of Hedyotis diffusa Wild; CRC, colorectal cancer; MTT, 3-(4,5-dimethylthiazol-2-yl)-2,5-diphenyltetrazolium bromide; DMSO, dimethyl sulfoxide; PI3K, phosphatidylinositol-3-kinase; AKT, protein kinase B; 5-FU, 5-fluorouracil; DAPI, 4',6-diamidino-2-phenylindole

Key words: Hedyotis diffusa Willd, colorectal cancer, drug resistance, 5-fluorouracil, cell proliferation and apoptosis, PI3K/AKT pathway the PI3K/AKT signaling pathway and expression of phosphatase and tensin homolog (PTEN), PI3K, AKT and phosphorylated (p)-AKT were determined. EEHDW significantly reduced cell viability, inhibited cell colony formation and promoted apoptosis of HCT-8/5-FU cells. Furthermore, EEHDW significantly downregulated the expression of Bcl-2, cyclin D1 and CDK4 and upregulated the expression of Bax and p21. In addition, EEHDW inhibited the activation of the PI3K/AKT pathway by increasing expression of PTEN and suppressing the expression of PI3K and p-AKT. The present study provided the first direct evidence that EEHDW may overcome drug-resistance in human CRC cells by inhibiting PI3K/AKT signaling pathway and provides a basis for the improved therapeutic use of HDW in the clinical treatment of cancer.

\section{Introduction}

Colorectal cancer (CRC) is one of the most common cancers worldwide, with over 1 million new cases diagnosed annually, along with 600,000 CRC-associated deaths (1). The 5-year survival rate for patients with early stage CRC is $\sim 80 \%$ (2). Current guidelines for the treatment of CRC are based on the stages of tumor progression. Surgery still remains the primary treatment option for $\mathrm{CRC}$, in conjunction with radiation therapy or chemotherapy.

The chemotherapeutic drug 5-fluorouracil (5-FU) is fundamental for the treatment of CRC. Patients with advanced stages of CRC are often have treated with a combination of various chemotherapeutic agents, including 5-FU, capecitabine, irinotecan, oxaliplatin, bevacizumab cetuximab, and panitumumab $(3,4)$. However, $\sim 40-50 \%$ of patients with advanced stage CRC may relapse or succumb due to the reduced efficacy of chemotherapy as a result of drug resistance. The mechanisms of cancer cell drug resistance involve complex systems, including increased drug efflux, reduced drug uptake, altered metabolism of drugs, altered expression of drug targets, reduced affinity of drug targets, activation of detoxification system, enhanced repair of drug-induced defects and resistance to apoptosis (5-7). Therefore, discovery of novel and more effective therapeutic agents which may reverse cancer cell drug resistance and increase clinical efficacy of CRC treatment is urgently required. 
Genetic abnormalities of the phosphatidylinositol-3-kinase $(\mathrm{PI} 3 \mathrm{~K})$ /protein kinase B (AKT) pathway are a common aspect of various cancers $(8,9)$. In addition, previous studies have revealed that the PI3K/AKT signaling pathway is involved in the development of cancer cell drug-resistance. PI3K is a lipid kinase that generates the secondary messenger lipid phosphatidylinositol (3-5)-triphosphate (PIP3), which in turn recruits and activates various proteins including AKT, a serine/threonine kinase $(10,11)$. Phosphorylation of AKT (p-AKT) mediates the activation of various downstream target genes involved in the regulation of cell proliferation, survival, angiogenesis, metastasis and drug resistance (12). The PI3K/AKT pathway may be involved in the regulation of a number of cellular processes, including cell death and survival, protein synthesis and metabolism (13). The activation of the PI3K/AKT signaling pathway and dysregulation of apoptosis are the major factors involved in the chemo-resistance of cancer cells by conferring acquired resistance to various chemotherapeutic drugs (14). Therefore, AKT is a novel target for the development of therapeutic drugs which may improve the outcomes of cancer chemotherapy.

Traditional Chinese Medicine (TCM) has been used for the treatment of various illnesses and diseases for thousands of years. Previous studies reported beneficial effects and reduced side effects of TCM in increasing the efficacy of cancer treatment, especially in combination with standard chemotherapeutic drugs (15-17). Therefore, the use of TCM promising when treating cancer cell drug-resistance and may allow for improved treatment of cancer. Hedyotis diffusa Willd (HDW) is a major component of TCM with potent anti-cancer effects on various cancers, such as ovarian, hepatocellular and cervical cancer (18-21). Previous studies have also demonstrated that HDW may inhibit CRC cell proliferation (22-26). These studies primarily focused on the effect of EEHDW on regular CRC cells. Recently, the present study reported that ethanol extract of HDW (EEHDW) may reduce 5-FU resistance in CRC HCT-8/5-FU cells by regulating the expression of permeability-glycoprotein and ATP binding cassette subfamily G member 2 (ABCG2) (27). However, the underlying mechanism of EEHDW overcoming drug resistance in human CRC HCT-8/5-FU cells remains to be fully elucidated. The present study aimed to investigate the inhibitory mechanism of EEHDW in terms of proliferation and apoptosis of CRC cells via regulation of the PI3K/AKT pathway.

\section{Materials and methods}

Materials and reagents. RPMI-1640 medium, fetal bovine serum (FBS), penicillin-streptomycin, trypsin-EDTA, TRIzol reagent, and bicinchoninic acid (BCA) protein assay kit, RIPA cell lysis buffer (pierce, 89,900) were obtained from Thermo Fisher Scientific, Inc. (Waltham, MA, USA). 5-FU, DMSO (cat. no. D5879), and 3-(4,5-dimethylthiazol-2-yl)-2,5-diphenyltetrazolium bromide (MTT; cat. no. M-2128) were obtained from Sigma-Aldrich; Merck Millipore (Darmstadt, Germany). Annexin V/propidium iodide (PI) apoptosis assay kit and DAPI were purchased from Nanjing KeyGen Biotech Co., Ltd. (Nanjing, China). PrimeScript ${ }^{\mathrm{TM}}$ RT Reagent kit was purchased from Takara Bio, Inc. (Otsu, Japan). Goldview Nucleic Acid Gel stain (cat. no. G8142) was purchased from Beijing Solarbio Life Science and Technology, Ltd., (Beijing, China). Primary antibodies for $\beta$-actin (cat.no.4967), B cell leukemia/lymphoma (Bcl-2; cat. no. 4223), Bcl-2 associated X (Bax; cat. no. 5023), cyclin D1 (cat. no. 2978), cyclin dependent kinase 4 (CDK4; cat. no. 2906), p21 (cat. no. 2947), PI3K (cat. no. 4257), p-AKT (Ser473; cat. no. 4060), AKT (cat. no. 2938) and phosphatase and tensin homolog (PTEN; cat. no. 9559), and horseradish peroxidase (HRP)-conjugated secondary antibodies (cat. no. 7074) were obtained from Cell Signaling Technology, Inc. (Danvers, MA, USA).

EEHDW preparation. HDW was purchased from a commercial supplier (Guo Yi Tang Chinese Herbal Medicine Store, Fujian, China) and the EEHDW was obtained as previously described (22). Stock solutions of EEHDW were prepared by dissolving $500 \mathrm{mg}$ EEHDW powder in $1 \mathrm{ml}$ DMSO to a final concentration of $500 \mathrm{mg} / \mathrm{ml}$ and stored at $-20^{\circ} \mathrm{C}$. Working solutions of EEHDW were made by diluting the stock solution in RPMI-1640 culture medium. The final concentration of DMSO in the medium was $<0.5 \%$.

Cell culture. Human colon carcinoma HCT-8/5-FU cell line was obtained from Nanjing KeyGen Biotech Co., Ltd. HCT-8/5-FU cells were cultured RPMI-1640 media supplemented with $10 \% \mathrm{FBS}$ and $100 \mathrm{U} / \mathrm{ml}$ penicillin and $100 \mathrm{~g} / \mathrm{ml}$ streptomycin in a humidified $37^{\circ} \mathrm{C}$ incubator supplemented with $5 \% \mathrm{CO}_{2}$.

Evaluation of cell viability of HCT-8/5-FU by MTT assay. Cell viability was determined using the MTT and colorimetric assay. Briefly, HCT-8/5-FU cells were seeded into 96-well plates at a density of $1.0 \times 10^{4}$ cells/well. After $24 \mathrm{~h}$, the cells were treated with different doses of EEHDW $(0.5-2.0 \mathrm{mg} / \mathrm{ml})$ for different periods of time (24 and $48 \mathrm{~h}$ ). Treatment with $0.1 \%$ DMSO was included as the vehicle control. Following treatment, $100 \mu \mathrm{l}$ MTT $(0.5 \mathrm{mg} / \mathrm{ml})$ were added to each well and cells were incubated for an additional $4 \mathrm{~h}$ at $37^{\circ} \mathrm{C}$. Subsequently, the MTT formazan precipitate was dissolved in $100 \mu \mathrm{l}$ DMSO and the absorbance was measured at $570 \mathrm{~nm}$ using an ELISA plate reader (Model EXL800, BioTek Instruments, Inc., Winooski, VT, USA).

Colony formation. HCT-8/5-FU cells from exponentially growing cultures were seeded into 12 -well culture plates at a density of $1.0 \times 10^{5}$ cells/well and were treated with different concentrations of EEHDW for $24 \mathrm{~h}$. Cells were then harvested and seeded into 6-well plates at a final density of $1.0 \times 10^{3}$ cells/well in $2 \mathrm{ml}$ fresh RPMI-1640 medium. Following incubation for 8 days, colonies were fixed in $\mathrm{MeOH}-\mathrm{HAc}$ (3:1, $\mathrm{v} / \mathrm{v}$ ) for $10 \mathrm{~min}$ in room temperature, stained with $0.1 \%$ crystal violet (Beyotime Biotech Co., Ltd. (Shanghai, China) for $10 \mathrm{~min}$ at room temperature and counted. Cell colony formation was calculated by showing survival of the control cells as $100 \%$.

Detection of apoptosis by flow cytometry analysis with Annexin V/PI staining and DAPI staining assay. HCT-8/5-FU cells at a density of $2.0 \times 10^{5}$ cells/well were seeded into 6-well plates and treated with different concentrations of EEHDW for $24 \mathrm{~h}$. Subsequently, apoptosis of HCT-8/5-FU cells was determined by flow cytometry analysis using a fluorescence 
activated cell sorting (FACS) caliber (BD Biosciences, Franklin Lakes, NJ, USA) and Annexin V-fluorescein isothiocyanate/PI kit, according to the manufacturer's protocol. Annexin V-negative/PI-negative cells indicated viable cells, whereas Annexin V-positive/PI-negative and Annexin V-positive/PI-positive cells indicated cells undergoing early and late stage apoptosis, respectively.

HCT-8/5-FU cell morphology and apoptosis was monitored following staining with DAPI. HCT-8/5-FU cells were seeded on 12-mm diameter round, glass cover slips in 24-well plates and treated with EEHDW $(0.5,1.0,2.0 \mathrm{mg} / \mathrm{ml})$ for $24 \mathrm{~h}$. The cover slips were then washed with PBS, fixed with $4 \%$ paraformaldehyde for $10 \mathrm{~min}$ and stained with DAPI $(4 \mu \mathrm{g} / \mathrm{ml})$ for $10 \mathrm{~min}$ at room temperature, then observed under fluorescence microscopy. Cells with clear condensed nuclei were identified as apoptotic cells.

$R N A$ extraction and reverse transcription-polymerase chain reaction (RT-PCR) analysis. HCT-8/5-FU cells were seeded into 6-well plates at a density of $4 \times 10^{5}$ cells/well and treated with different concentrations of EEHDW for $24 \mathrm{~h}$. RNA was extracted from HCT-8/5-FU cells using TRIzol reagent (Takara Bio, Inc.). cDNA was obtained using reverse transcription with PrimeScript RT reagent kit, according to the manufacturer's protocol. PCR was performed to determine the mRNA expression levels of Bax, Bcl-2, cyclin D1, CDK4 and p21. GAPDH was used as an internal control. The primers used for amplification of Bax, Bcl-2, cyclin D1, CDK4 and p21 transcripts are as follows: GAPDH forward (F) 5'-GTCATCCATGAC AACTTTGG-3' and reverse (R) 5'-GAGCTTGACAAAGTG GTCGT-3'; Bcl-2 F 5'-CAGCTGCACCTGACGCCCTT-3' and R 5'-GCCTCCGTTATCCTGGATCC-3'; Bax F 5'-TGC TTCAGGGTTTCATCCAGG-3' and R 5'-TGGCAAAGT AGAAAAGGG CGA-3'; CDK4 F 5'-CATGTAGACCAGGAC CTAAGC-3' and R 5'-AACTGGCGCATCAGATCCTAG-3'; cyclin Dl F 5'-TGGATGCTGGAGGTCTGCGAGGAA-3' and R 5'-GGCTTCGATCTGCTCCTGGCAGGC-3'; p21 F 5'-GAGCGATGGAACTTCGACTTTGTC-3' and R 5'-GGC GTTTGGAGTGGTAGAAATCTG-3'. The PCR was repeated 3 independent times. A BIO-RAD S1000 Thermal Cycler (Bio-Rad Laboratories, Inc., Hercules, CA, USA) was used to perform the experiment under the following conditions: $95^{\circ} \mathrm{C}$ for $30 \mathrm{sec}$, annealing at the $60^{\circ} \mathrm{C}$ for $30 \mathrm{sec}$ and extension at $72^{\circ} \mathrm{C}$ for $30 \mathrm{sec}$ for 30 cycles. Samples were analyzed by $1.5 \%$ agarose gel electrophoresis. The DNA bands were examined using a gel documentation system (Gel Doc 2000; Bio-Rad Laboratories, Inc.). PCR results were calculated based on 3 independent experiments.

Western blot analysis. Western blot analysis was used to observe HCT-8/5-FU cell apoptosis induced by EEHDW treatment and expression of PI3K/AKT signaling pathways. HCT-8/5-FU cells were seeded into $25 \mathrm{~cm}^{2}$ flasks at a density of $1.0 \times 10^{6}$ cells/flask in $5 \mathrm{ml}$ RPMI-1640 media and treated with different concentrations of EEHDW for $24 \mathrm{~h}$. Cells were lysed using RIPA cell lysis buffer containing protease inhibitors, and the resulting protein concentration in each sample was determined by BCA assay. Equal quantities of protein $(50 \mu \mathrm{g})$ were then separated on 10\% SDS-PAGE gel and transferred onto PVDF membranes. The membranes were blocked for $2 \mathrm{~h}$ with $5 \%$ non-fat dry milk at room temperature, then incubated with $\beta$-actin, Bax, Bcl-2, Cyclin D1, CDK4, p21, PI3K, p-AKT, AKT and PTEN primary antibodies $(1: 1,000)$ overnight at $4^{\circ} \mathrm{C}$. Following washing and subsequent incubation with HRP-conjugated secondary antibodies $(1: 2,000)$ for $2 \mathrm{~h}$ at room temperature, protein bands of interest were detected using enhanced chemiluminescence by SuperSignal West Pico Chemiluminescent Substrate. Image $\mathrm{Lab}^{\mathrm{TM}}$ software version 3.0 (Bio-Rad Laboratories, Inc.) was used for densitometric analysis and quantification of western blots.

Statistical analysis. Data were expressed as the mean \pm standard deviation and analyzed using SPSS version 17.0 (SPSS, Inc., Chicago, IL, USA) by using Student's t-test or one-way AVOVA analysis. LSD and Dunnet's were used as post-hoc tests. $\mathrm{P}<0.05$ was considered to indicate a statistically significant difference.

\section{Results}

EEHDW reduces HCT-8/5-FU cell viability. HCT-8/5-FU cell viability was measured using MTT assay following exposure to different concentrations of EEHDW for $24 \mathrm{~h}$ and $48 \mathrm{~h}$. As presented in Fig. 1, treatment with $0.5-2.0 \mathrm{mg} / \mathrm{ml}$ of EEHDW for 24 and $48 \mathrm{~h}$ significantly reduced cell viability of HCT-8/5-FU cells by $22.22-47.58 \%$ and $26.67-78.27 \%$, respectively compared with untreated control cells $(\mathrm{P}<0.01$; Fig. 1). This suggests that EEHDW inhibited HCT-8/5-FU cell viability in a concentration- and time-dependent manner.

EEHDW inhibits the colony formation ability of HCT-8/5-FU cells. In order to fully evaluate the effect of EEHDW on HCT-8/5-FU cells, the number of colonies formed by HCT-8/5-FU cells following EEHDW treatment was examined using a colony formation assay. As presented in Fig. 2, treatment with $0.5,1.0$ and $2.0 \mathrm{mg} / \mathrm{ml}$ EEHDW for $24 \mathrm{~h}$ significantly reduced the cell survival rate of HCT-8/5-FU cells when compared with untreated control cells in a dose-dependent manner $(\mathrm{P}<0.05)$.

EEHDW induces apoptosis in HCT-8/5-FU cells. Due to the innate resistance of the cancer cells to apoptosis, clinical treatment for various cancers such as CRC is frequently insufficient. The present study examined the possibility that EEHDW may overcome drug-resistance and induce apoptosis of HCT-8/5-FU cells. Annexin-V/PI staining and FACS analysis demonstrated that EEHDW treatment significantly increased the percentage of HCT-8/5-FU cells undergoing early-stage (lower right quadrant) and late-stage apoptosis (upper right quadrant) in a dose-dependent manner when compared with the untreated control cells (Fig. $3 \mathrm{~A}$ and $\mathrm{B} ; \mathrm{P}<0.05$ ). The cellular and nuclear morphology of HCT-8/5-FU cells was examined using DAPI staining. As presented in Fig. 3C, EEHDW treated cells showed more intense staining and apparent DNA condensation when compared with untreated control cells, suggesting that EEHDW treatment promoted HCT-8/5-FU cell apoptosis.

EEHDW affects the expression of cyclin D1, CDK4, p21, $B c l-2$ and Bax in HCT-8/5-FU cells. The proliferation of the majority of animal cells is primarily regulated in the G1/S 


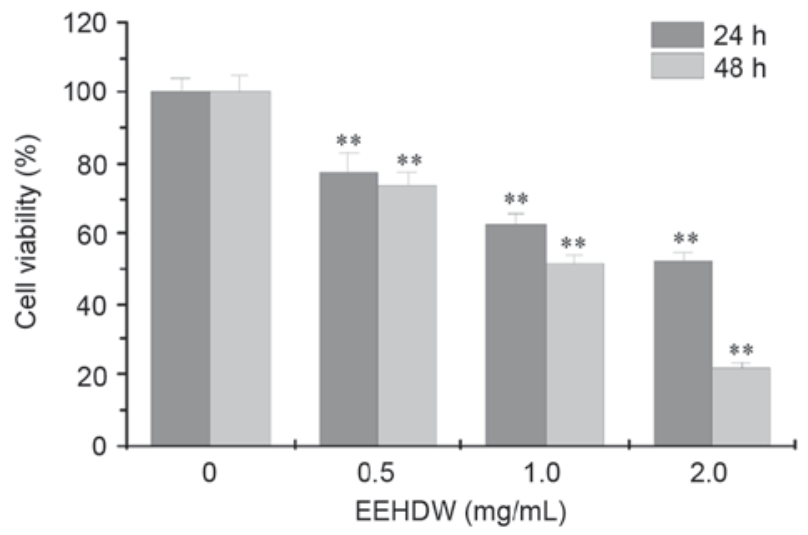

Figure 1. Effect of EEHDW on cell viability of HCT-8/5-FU cells HCT-8/5-FU cells were treated with different concentrations of EEHDW for 24 and $48 \mathrm{~h}$. Cell viability was determined using MTT assay. The data were normalized to the viability of control cells. Data are presented as mean \pm standard deviation of three independent experiments. ${ }^{* *} \mathrm{P}<0.01$ vs. untreated control cells. EEHDW, ethanol extract of Hedyotis diffusa Willd; 5-FU, 5-fluorouracil

transition, one of the two main checkpoints in the cell cycle which is mediated by the pro-proliferative cyclin D1 and CDK4. Apoptosis is tightly regulated by Bcl-2 family proteins, including anti-apoptotic members such as Bcl-2 and pro-apoptotic members such as Bax (28-30). In order to investigate the underlying mechanisms of EEHDW and how it overcomes the drug resistance of cancer cells, the present study examined the mRNA and protein expression levels of cyclin D1, CDK4, p21, Bax and Bcl-2 following EEHDW treatment in HCT-8/5-FU cells. As presented in Fig. 4, EEHDW treatment significantly reduced cyclin D1, CDK4 and Bcl-2 mRNA and protein expression; however, $\mathrm{p} 21$ and Bax mRNA and protein expression levels in HCT-8/5-FU cells were increased when compared with untreated control cells $(\mathrm{P}<0.05)$. This suggests that EEHDW likely modulates the drug resistance of CRC HCT-8/5-FU cells by regulating the expression of cyclin D1, CDK4, p21, Bcl-2 and Bax.

EEHDW inhibits the activation of the PI3K/AKT pathway in HCT-8/5-FU cells. The PI3K/AKT signaling pathway has a crucial role in numerous cellular systems and its activation is closely associated with the prevention of cellular apoptosis. AKT is activated by phospholipid binding and activation loop phosphorylation at Thr308 by pyruvate dehydrogenase kinase 1 and by phosphorylation within the carboxy terminus at Ser473. In order to determine the mechanism behind EEHDW's ability to overcome drug resistance of CRC, the present study examined the expression of key proteins involved in the PI3K/AKT signaling pathway, including PI3K, p-AKT, AKT and PTEN following EEHDW treatment. As presented in Fig. 5, EEHDW treatment significantly reduced PI3K and p-AKT expression and the ratio of p-AKT to total AKT; however, led to increased PTEN expression in HCT-8/5-FU cells, in a dose-dependent manner when compared with untreated control cells $(\mathrm{P}<0.05)$. This suggests that EEHDW may also modulate the drug-resistance of CRC HCT-8/5-FU cells via regulation of the PI3K/AKT signaling pathway.
A
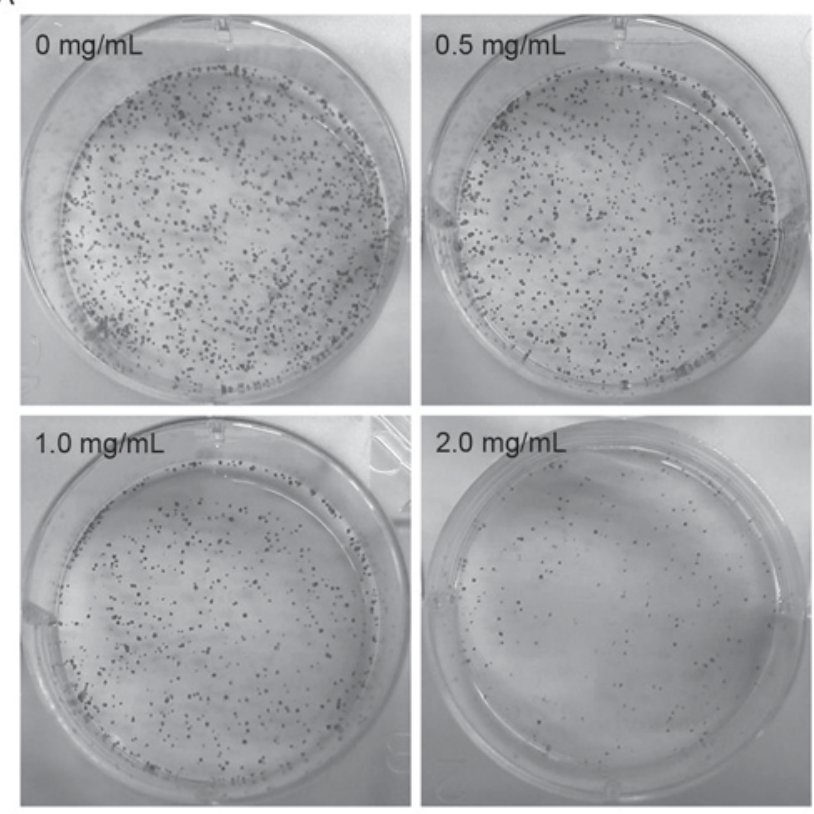

B

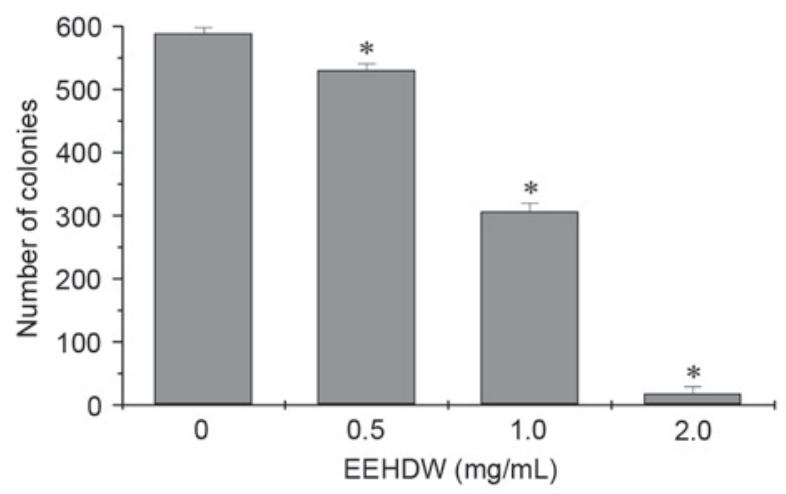

Figure 2. Effect of EEHDW on HCT-8/5-FU cell colony formation. (A) HCT-8/5-FU cells were treated with the indicated concentrations of EEHDW for $24 \mathrm{~h}$. cells were seeded at a density of $1 \times 10^{3}$ cells/well into 6 -well plates and incubated for 8 days. Formed colonies were fixed in $\mathrm{MeOH}-\mathrm{HAc}(3: 1, \mathrm{v} / \mathrm{v})$ for $10 \mathrm{~min}$, stained with crystal violet and counted. (B) Data are presented as mean \pm standard deviation of three independent experiments. "P<0.05 vs. untreated control cells. EEHDW, ethanol extract of Hedyotis diffusa Willd; 5-FU, 5-fluorouracil.

\section{Discussion}

EEHDW is a major component commonly used in traditional Chinese medicine for the clinical treatment of CRC. Our previous study determined that Hedyotis diffusa Willd inhibits the growth of CRC, possibly via the inhibition of tumor angiogenesis by regulating the Hedgehog signaling pathway (25). It also induced the cell apoptosis via the IL-6-inducible STAT3 pathway (26). However, TCM including HDW have multiple components. There are various compounds in EEHDW such as ursolic and oleanolic acid, kaempferol, luteolin and they may regulate multiple signaling pathways (31). Our previous study revealed that EEHDW significantly reduced the viability of CRC HCT-8/5-FU cells by inhibiting cell proliferation and inducing cell apoptosis (27). A previous study demonstrated that EEHDW may restore the sensitivity of multi-drug resistant cancer cells to various chemotherapeutic agents (32). 
A
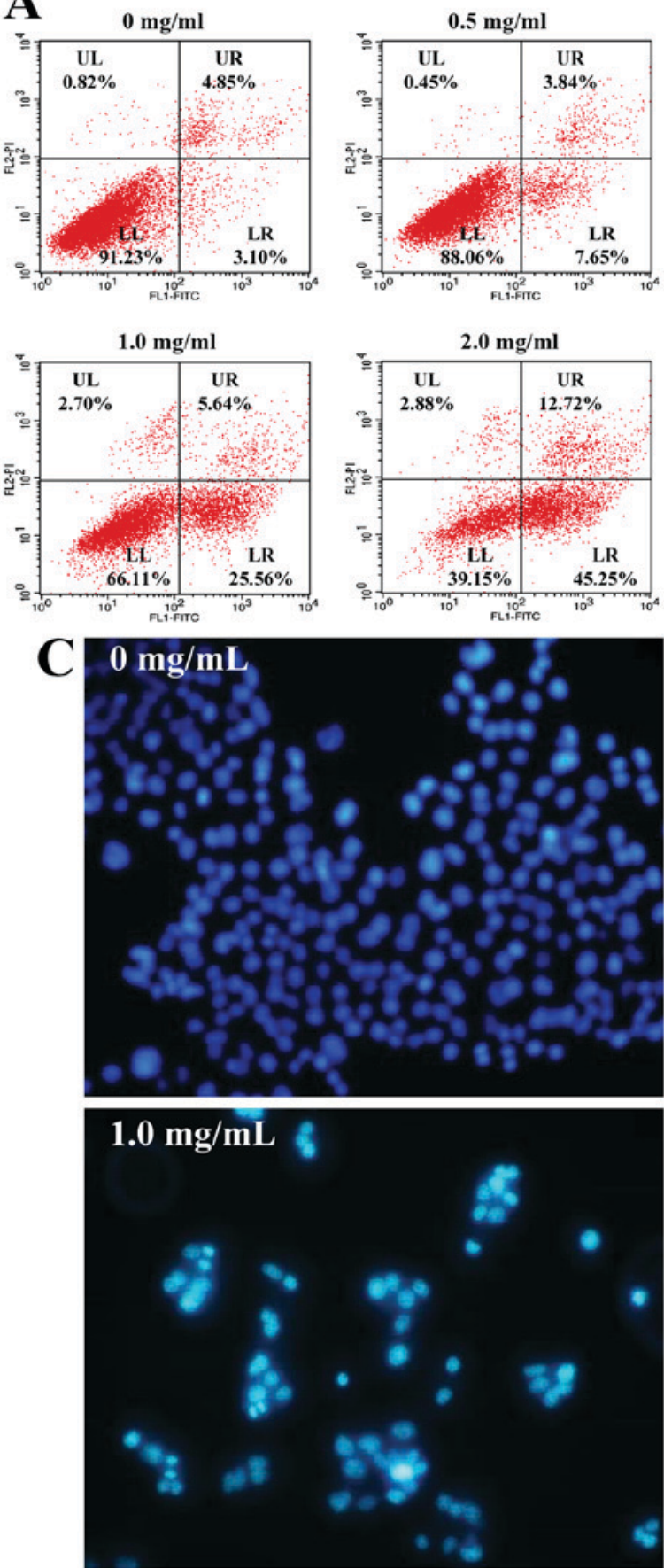

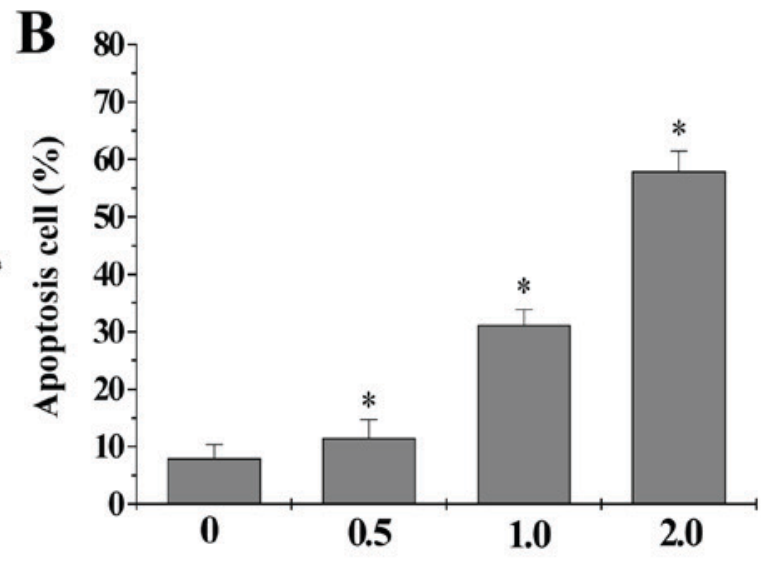

EEHDW $(\mathrm{mg} / \mathrm{ml})$
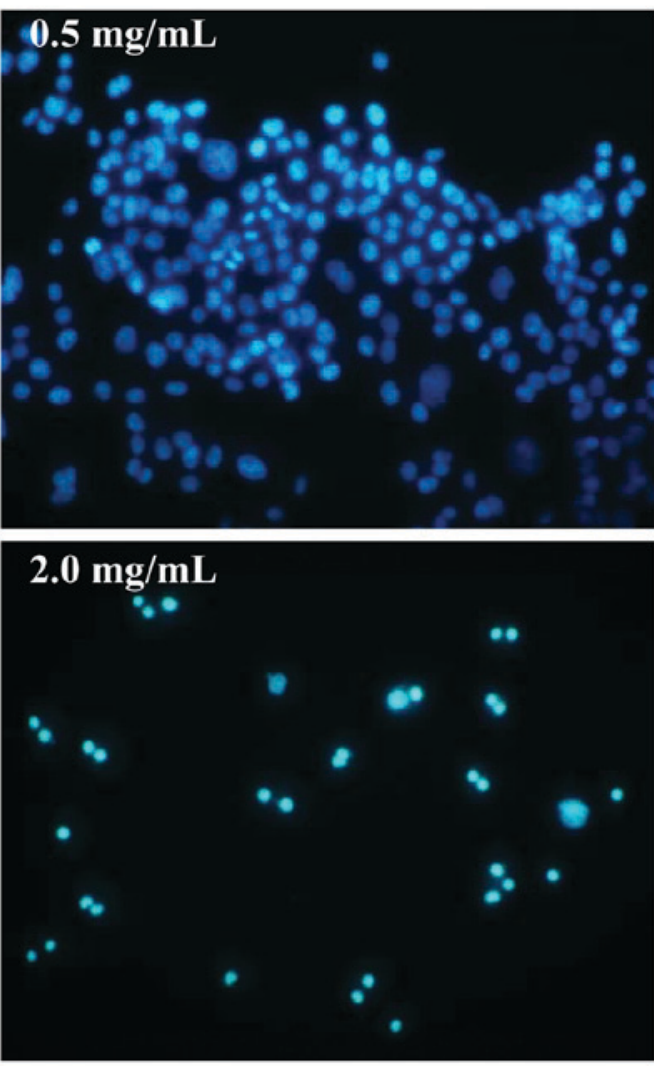

Figure 3. Effect of EEHDW in inducing apoptosis of HCT-8/5-FU cells. (A) Cells were treated with various concentration of EEHDW for 24 h prior to Annexin V/PI staining and FACS analysis. Double-negative stained cells indicate live cell population; Annexin V-positive/PI-negative stained cells and Annexin V/PI double-positive stained cells represent early apoptotic and late apoptotic cells, respectively; Annexin V-negative and PI-positive stained cells represent dead cells. (B) Quantification of FACS analysis. Data are presented as mean \pm standard deviation of three independent experiments. "P $<0.05$ vs. untreated control cells. (C) HCT-8/5-FU cells were treated with the indicated dosages of EEHDW for $24 \mathrm{~h}$. Changes in cellular morphology were visualized by staining with DAPI and observed under a phase-contrast microscope. Magnification, x200. Images are representative of three independent experiments. EEHDW, ethanol extract of Hedyotis diffusa Willd; PI, propidium iodide; FACS, fluorescence activated cell sorting; FITC, fluorescein isothiocyanate; 5-FU, 5-fluorouracil.

However, the mechanisms of drug-resistance in cancer cells involve complex systems, including increased drug efflux, reduced drug uptake and resistance to apoptosis (5-7). Our previous study demonstrated that EEHDW is effective for increasing 5-FU accumulation in HCT-8/5-FU cells and that EEHDW may reverse 5-FU resistance by inhibiting the expression of an $\mathrm{ABC}$ transporter protein, ABCG2 (27). In addition, resistance to apoptosis has been implicated as a major factor involved in chemo-resistance of cancer cells. The present study aimed to elucidate the mechanisms of EEHDW in inhibiting proliferation and inducing apoptosis in CRC cells via regulation of the PI3K/AKT pathway.

The current study demonstrated that the growth and viability of CRC HCT-8/5-FU cells was significantly inhibited by EEHDW treatment, through inhibition of cell proliferation and induction of cellular apoptosis. One of the hallmarks of 
A

$\operatorname{EEHDW}(\mathrm{mg} / \mathrm{mL})$
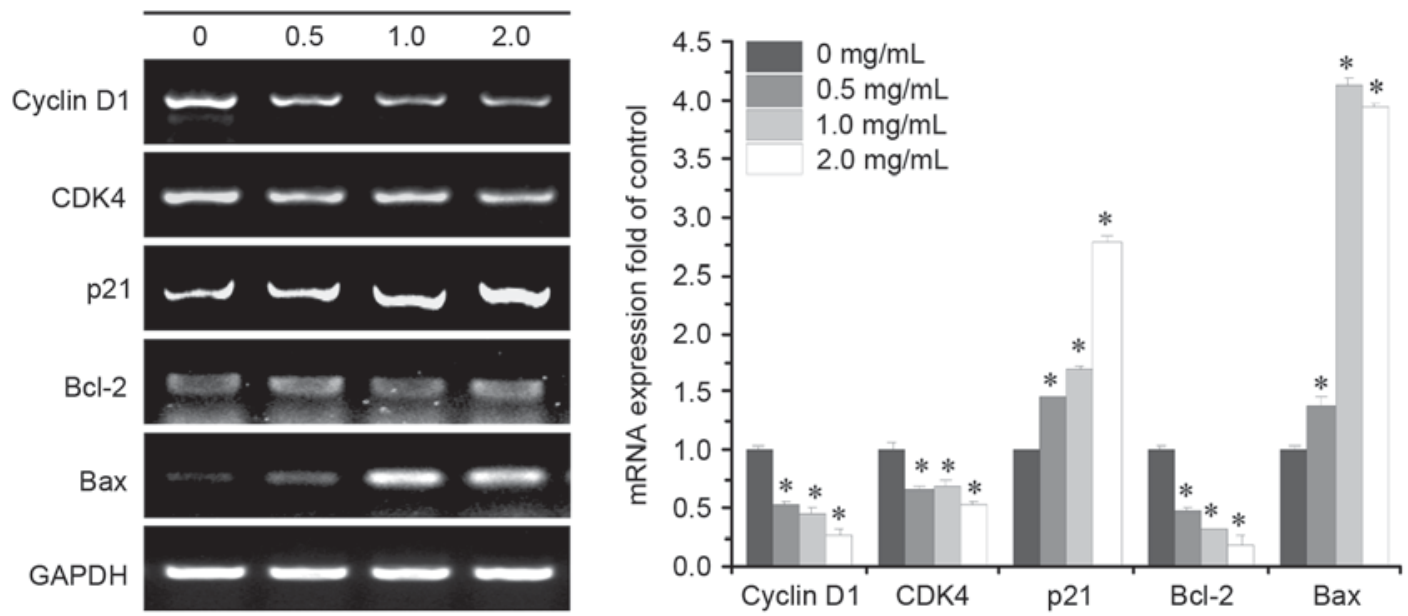

B $\operatorname{EEHDW}(\mathrm{mg} / \mathrm{mL})$
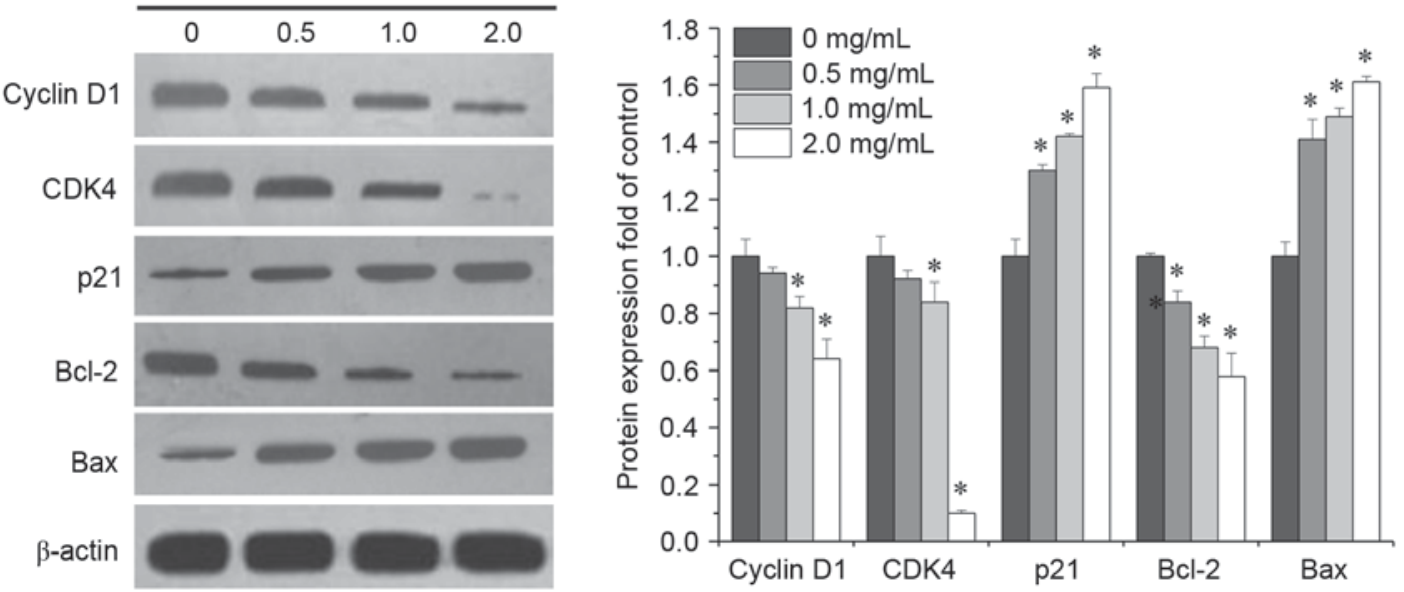

Figure 4. Effect of EEHDW on the mRNA and protein expression levels of cyclin D1, CDK4, p21, Bcl-2 and Bax in HCT-8/5-FU cells. HCT-8/5-FU cells were treated with the indicated concentration of EEHDW for $24 \mathrm{~h}$. The mRNA (A) expression levels of cyclin D1, CDK4, p21, Bcl-2, and Bax were subsequently determined by using reverse transcription-polymerase chain reaction. Densitometric analysis was conducted to indicated the mRNA expression fold change. The data were normalized to the mean mRNA expression of untreated control. The protein (B) expression levels of cyclin D1, CDK4, p21, Bcl-2, and Bax were subsequently determined by western blot analysis. Densitometric analysis was conducted to indicated the protein expression fold-change. Data were normalized to the mean protein expression of untreated control (fold of control). " $\mathrm{P}<0.05$ vs. untreated control cells. CDK4, cyclin dependent kinase 4; Bcl-2, B cell leukemia/lymphoma 2; Bax, Bcl2 associated X, apoptosis regulator; EEHDW, ethanol extract of Hedyotis diffusa Willd; 5-FU, 5-fluorouracil.

A

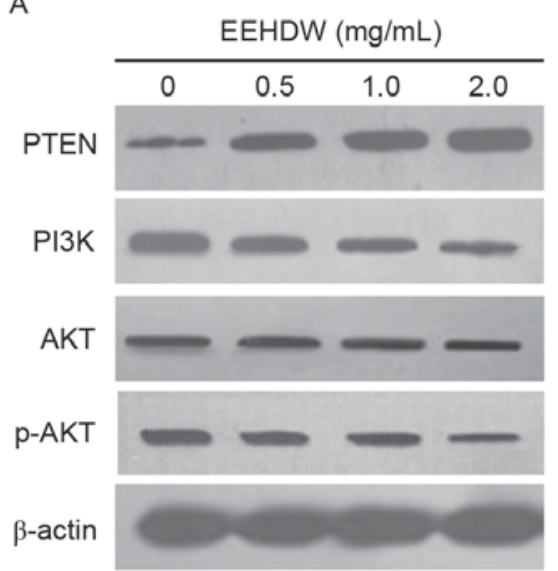

B

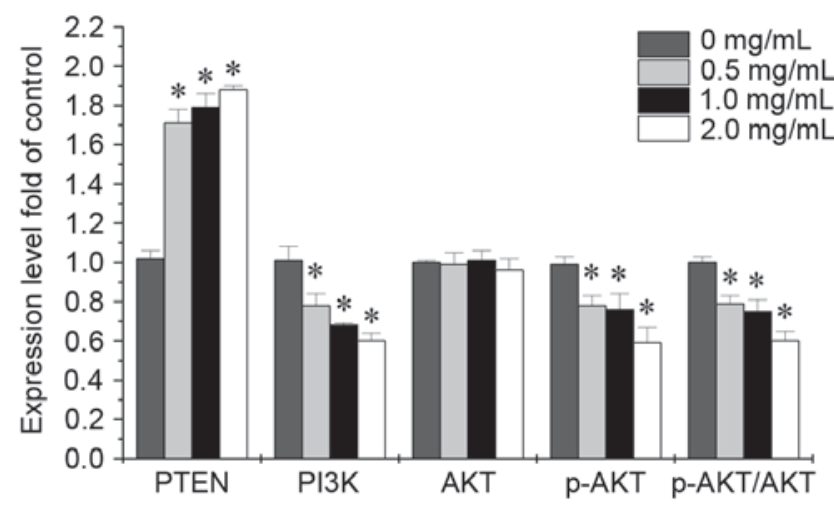

Figure 5. Effect of EEHDW on the activation of PI3K/AKT signaling pathway in HCT-8/5-FU cells. (A) Expressions of PTEN, PI3K, AKT and p-AKT following EEHDW treatment in HCT-8/5-FU cells were determined by western blot analysis. Data are representative of three independent experiments. (B) Densitometric analysis was conducted to indicated the protein expression fold-change. The data were normalized to the mean protein expression of untreated control. "P<0.05 vs. untreated control cells. EEHDW, ethanol extract of Hedyotis diffusa Willd; PTEN, phosphatase and tensin homolog; PI3K, phosphoinositide 3-kinase; AKT, protein kinase B; p-AKT, phosphorylated-AKT; 5-FU, 5-fluorouracil. 
cancerous cells is their ability to resist the intrinsic process of cellular apoptosis (33). Eliminating or reducing the ability of cancer cells to resist apoptosis has become a key target for anti-cancer therapy (34). Therefore, determining the underlying mechanisms involved in cell apoptotic pathways has become a crucial step in the clinical treatment of cancer (35). Additionally, cancer cells have the ability to upregulate the expression of anti-apoptotic regulators such as Bcl-2, whilst downregulating pro-apoptotic factors such as Bax $(36,37)$. The present study demonstrated that EEHDW may induce apoptosis of HCT-8/5-FU cells and restore its sensitivity to chemotherapy. In addition, the present study determined that EEHDW treatment significantly reduced the expression of Bcl-2, cyclin D1 and CDK4, whilst significantly increasing the expression of Bax and p21, demonstrating that EEHDW treatment may suppress proliferation and induce apoptosis of HCT-8/5-FU cells.

The PI3K/AKT signaling pathway is involved in the promotion of cell proliferation and apoptosis $(10,38)$. Previous studies have revealed that inhibition of the PI3K/AKT pathway may restore the sensitivity of various cancer cells to chemotherapeutic drugs (38-40). In particular, p-AKT activation leads to the increased transcription of downstream target genes such as Bax, Bcl-2, CDK4, cyclin D1, ATP binding cassette subfamily B member 1 and ABCG2 $(16,17,41)$, which are associated with the regulation of various cellular processes including cell cycle, proliferation and apoptosis. Therefore, the PI3K/AKT signaling pathway and its downstream genes are promising targets for the therapeutic treatment of cancer (42). The present study detected a significant increase in protein expression of PTEN and reduction in the protein expression of PI3K or p-AKT following EEHDW treatment in HCT-8/5-FU cells, demonstrating that EEHDW may overcome the drug-resistance of cancer cells via inhibition of the PI3K/AKT pathway. Therefore, the present study outlined the key anti-drug resistance effect of EEHDW on CRC HCT-8/5-FU cells.

In conclusion, the current study demonstrated that EEHDW treatment may inhibit proliferation and induce apoptosis of 5-FU resistant CRC cells via inhibition of p-AKT activation and regulation of Bcl-2, Bax, cyclin D1, CDK4 and p21 expression. Additionally, the present study demonstrated that regulation of the PI3K/AKT pathway and its downstream target genes is a key mechanism of EEHDW in overcoming 5-FU cancer drug resistance.

\section{Acknowledgements}

The present study was sponsored by the Research Fund for the Doctoral Program of Higher Education of China (grant no. 20133519110003), Project Funding for the Training of Young and Middle-aged Backbone Personnel of Fujian Provincial Health and Family Planning Commission (grant no. 2016-ZQN-67) and the Developmental Fund of Chen Keji Integrative Medicine (grant nos. CKJ2014013 and CKJ2015007).

\section{References}

1. Jemal A, Bray F, Center MM, Ferlay J, Ward E and Forman D: Global cancer statistics. CA Cancer J Clin 61: 69-90, 2011.

2. Siegel R, Naishadham D and Jemal A: Cancer statistics, 2013. CA Cancer J Clin 63: 11-30, 2013.
3. Van Cutsem E, Nordlinger B and Cervantes A; ESMO Guidelines Working Group: Advanced colorectal cancer: ESMO clinical practice guidelines for treatment. Ann Oncol 21 (Suppl 5): v93-v97. 2010.

4. Tournigand C, André T, Achille E, Lledo G, Flesh M, Mery-Mignard D, Quinaux E, Couteau C, Buyse M, Ganem G, et al: FOLFIRI followed by FOLFOX6 or the reverse sequence in advanced colorectal cancer: A randomized GERCOR study. J Clin Oncol 22: 229-237, 2004.

5. Li W, Zhang H, Assaraf YG, Zhao K, Xu X, Xie J, Yang DH and Chen ZS: Overcoming ABC transporter-mediated multidrug resistance: Molecular mechanisms and novel therapeutic drug strategies. Drug Resist Updat 27: 14-29, 2016.

6. Mittal B, Tulsyan S, Kumar S, Mittal RD and Agarwal G: Cytochrome P450 in cancer susceptibility and treatment. Adv Clin Chem 71: 77-139, 2015.

7. Pan ST, Li ZL, He ZX, Qiu JX and Zhou SF: Molecular mechanisms for tumour resistance to chemotherapy. Clin Exp Pharmacol Physiol 43: 723-737, 2016.

8. Bartholomeusz $\mathrm{C}$ and Gonzalez-Angulo AM: Targeting the PI3K signaling pathway in cancer therapy. Expert Opin Ther Targets 16: 121-130, 2012.

9. Engelman JA: Targeting PI3K signalling in cancer: Opportunities, challenges and limitations. Nat Rev Cancer 9: 550-562, 2009

10. Courtney KD, Corcoran RB and Engelman JA: The PI3K pathway as drug target in human cancer. J Clin Oncol 28: 1075-1083, 2010.

11. Song L, Xiong H, Li J, Liao W, Wang L, Wu J and Li M: Sphingosine kinase-1 enhances resistance to apoptosis through activation of PI3K/AKT/NF-kB pathway in human non-small cell lung cancer. Clin Cancer Res 17: 1839-1849, 2011.

12. Housman G, Byler S, Heerboth S, Lapinska K, Longacre M, Snyder N and Sarkar S: Drug resistance in cancer: An overview. Cancers (Basel) 6: 1769-1792, 2014.

13. Danielsen SA, Eide PW, Nesbakken A, Guren T, Leithe E and Lothe RA: Portrait of the PI3K/AKT pathway in colorectal cancer. Biochim Biophys Acta 1855: 104-121, 2015.

14. Brotelle T and Bay JO: PI3K-AKT-mTOR pathway: Description, therapeutic development, resistance, predictive/prognostic biomarkers and therapeutic applications for cancer. Bull Cancer 103: 18-29, 2016 (In French).

15. Xu D, Lu Q and Hu X: Down-regulation of P-glycoprotein expression in MDR breast cancer cell MCF-7/ADR by honokiol. Cancer Lett 243: 274-280, 2006.

16. Wang J, Xia Y, Wang H and Hou Z: Chinese herbs of Shenghe Powder reverse multidrug resistance of gastric carcinoma SGC-7901. Integr Cancer Ther 6: 400-404, 2007.

17. Angelini A, Di Ilio C, Castellani ML, Conti P and Cuccurullo F: Modulation of multidrug resistance p-glycoprotein activity by flavonoids and honokiol in human doxorubicin- resistant sarcoma cells (MES-SA/DX-5): Implications for natural sedatives as chemosensitizing agents in cancer therapy. J Biol Regul Homeost Agents 24: 197-205, 2010.

18. Zhang L, Zhang J, Qi B, Jiang G, Liu J, Zhang P, Ma Y and $\mathrm{Li} \mathrm{W}$ : The anti-tumor effect and bioactive phytochemicals of Hedyotis diffusa willd on ovarian cancer cells. J Ethnopharmacol 192: 132-139, 2016.

19. Li YL, Zhang J, Min D, Hongyan Z, Lin N and Li QS: Anticancer effects of 1,3-dihydroxy-2-methylanthraquinone and the ethyl acetate fraction of Hedyotis diffusa willd against HepG2 carcinoma cells mediated via apoptosis. PLoS One 11: e0151502, 2016.

20. Zhang P, Zhang B, Gu J, Hao L, Hu F and Han C: The study of the effect of Hedyotis diffusa on the proliferation and the apoptosis of the cervical tumor in nude mouse model. Cell Biochem Biophys 72: 783-789, 2015.

21. Kuo YJ, Yang JS, Lu CC, Chiang SY, Lin JG and Chung JG: Ethanol extract of Hedyotis diffusa willd upregulates G0/G1 phase arrest and induces apoptosis in human leukemia cells by modulating caspase cascade signaling and altering associated genes expression was assayed by cDNA microarray. Environ Toxicol 30: 1162-1177, 2015.

22. Lin J, Chen Y, Wei L, Chen X, Xu W, Hong Z, Sferra TJ and Peng J: Hedyotis diffusa willd extract induces apoptosis via activation of the mitochondrion-dependent pathway in human colon carcinoma cells. Int J Oncol 37: 1331-1338, 2010.

23. Lin JM, Wei LH, Xu W, Hong Z, Liu X and Peng J: Effect of Hedyotis diffusa willd extract on tumor angiogenesis. Mol Med Rep 4: 1283-1288, 2011. 
24. Cai Q, Lin J, Wei L, Zhang L, Wang L, Zhan Y, Zeng J, Xu W, Shen A, Hong Z and Peng J: Hedyotis diffusa willd inhibits colorectal cancer growth in vivo via inhibition of STAT3 signaling pathway. Int J Mol Sci 13: 6117-6128, 2012.

25. Lin J, Wei L, Shen A, Cai Q, Xu W, Li H, Zhan Y, Hong Z and Peng J: Hedyotis diffusa willd extract suppresses sonic hedgehog signaling leading to the inhibition of colorectal cancer angiogenesis. Int J Oncol 42: 651-656, 2013.

26. Lin J, Li Q, Chen H, Lin H, Lai Z and Peng J: Hedyotis diffusa willd. Extract suppresses proliferation and induces apoptosis via IL-6-inducible STAT3 pathway inactivation in human colorectal cancer cells. Oncol Lett 9: 1962-1970, 2015.

27. Li Q, Wang X, Shen A, Zhang Y, Chen Y, Sferra TJ, Lin J and Peng J: Hedyotis diffusa Willd overcomes 5-fluorouracil resistance in human colorectal cancer HCT-8/5-FU cells by downregulating the expression of P-glycoprotein and ATP-binding casette subfamily G member 2. Exp Ther Med 10: 1845-1850, 2015.

28. Elledge SJ: Cell cycle checkpoints: Preventing an identity crisis. Science 274: 1664-1672, 1996.

29. Taulés M, Rius E, Talaya D, López-Girona A, Bachs O and Agell N: Calmodulin is essential for cyclin-dependent kinase $4(\mathrm{Cdk} 4)$ activity and nuclear accumulation of cyclin D1-Cdk4 during G1. J Biol Chem 273: 33279-33386, 1998.

30. Adams JM and Cory S: The Bcl-2 apoptotic switch in cancer development and therapy. Oncogene 26: 1324-1337, 2007.

31. Youle RJ and Strasser A: The Bcl-2 protein family: Opposing activities that mediate cell death. Nat Rev Mol Cell Biol 9: 47-59, 2008.

32. Chen XZ, Cao ZY, Chen TS, Zhang YQ, Liu ZZ, Su YT, Liao LM and Du J: Water extract of Hedyotis diffusa willd suppresses proliferation of human HepG2 cells and potentiates the anticancer efficacy of low-dose 5-fluorouracil by inhibiting the CDK2-E2F1 pathway. Oncol Rep 28: 742-748, 2012.

33. Hanahan D and Weinberg RA: Hallmarks of cancer: The next generation. Cell 144: 646-674, 2011.
34. Liu JJ, Lin M, Yu JY, Liu B and Bao JK: Targeting apoptotic and autophagic pathways for cancer therapeutics. Cancer Lett 300: 105-114, 2011.

35. Denicourt C and Dowdy SF: Medicine. Targeting apoptotic pathways in cancer cells. Science 305: 1411-1413, 2004.

36. Kang MH and Reynolds CP: Bcl-2 inhibitors: Targeting mitochondrial apoptotic pathways in cancer therapy. Clin Cancer Res 15: 1126-1132, 2009.

37. Tsuchiya T, Tsuno NH, Asakage M, Yamada J, Yoneyama S, Okaji Y, Sasaki S, Kitayama J, Osada T, Takahashi K and Nagawa H: Apoptosis induction by p38 MAPK inhibitor in human colon cancer cells. Hepatogastroenterology 55: 930-935, 2008.

38. Wu H, Hait WN and Yang JM: Small interfering RNA-induced suppression of MDR1 (P-glycoprotein) restores sensitivity to multidrug-resistant cancer cells. Cancer Res 63: 1515-1519, 2003.

39. Jiao $M$ and Nan KJ: Activation of PI3 kinase/Akt/HIF-1a pathway contributes to hypoxia-induced epithelial-mesenchymal transition and chemoresistance in hepatocellular carcinoma. Int J Oncol 40: 461-468, 2012.

40. Zhang HY, Zhang PN and Sun H: Aberration of the $\mathrm{PI} 3 \mathrm{~K} / \mathrm{AKT} / \mathrm{mTOR}$ signaling in epithelial ovarian cancer and its implication in cisplatin-based chemotherapy. Eur J Obstet Gynecol Reprod Biol 146: 81-86, 2009.

41. Lima RT, Martins LM, Guimarães JE, Sambade C and Vasconcelos MH: Specific downregulation of Bcl-2 and xIAP by RNAi enhances the effects of chemotherapeutic agents in MCF-7 human breast cancer cells. Cancer Gene Ther 11: 309-316, 2004.

42. Wong KK, Engelman JA and Cantley LC: Targeting the PI3K signaling pathway in cancer. Curr Opin Genet Dev 20: 87-90, 2010. 